\title{
Pseudo-Johannes Duns Scotus über Induktion
}

\section{§1. Einleitung}

I

nduktion > ist einer der wichtigsten traditionellen Grundbegriffe, um Wissenschaft zu rekonstruieren. Traditionell interpretierte man Induktion r als die Methode, mit der die Wissenschaft die Axiome und Gesetze auffindet. Empirische Gegebenheiten veranlassen den Geist des Wissenschaftlers, diese Axiome und Gesetze aufzustellen. Auch wenn in der modernen Wissenschaftstheorie, besonders seit den Schriften K.R. Poppers in der ersten Hälfte der 20. Jahrhunderts, Induktion` nicht mehr als einziger Grundbegriff bei dieser Rekonstruktion der Wissenschaft ausgezeichnet wird, spielt der Begriff immer noch eine prominente Rolle.

Man könnte auf eine moderne Weise Induktion, bestimmen als ein nicht-beweiskräftiges Argument, in welchem die Wahrheit der Prämissen nicht logisch die Wahrheit der Konklusion impliziert, aber eine gute Veranlassung gibt, der Konklusion Glauben zu schenken ${ }^{1}$. Zum Beispiel kann die Eigenschaft 'Ausdehnung' nach einigen Experimenten, bei denen Stücke von Eisen erwärmt worden sind, der natürlichen Art Eisen zugeschrieben werden.

Man könnte unterscheiden zwischen (vollkommener) und (wahrscheinlicher Induktion?. Das soeben beschriebene induktive Argument könnte man svollkommene Induktion' nennen zur Unterscheidung von einer 'Deduktion, in welcher logisch notwendig von Prämissen auf eine Konklusion geschlossen wird. Diese logische Induktion? ist nur logisch gültig, wenn eine allgemeine Aussage als Vermittler der Argumentation vorausgesetzt ist. Man hat aber nicht immer eine Garantie, daß diese universelle vermittelnde Aussage widerstandsfähig ist gegen Proben, denn diese allgemeine Aussage ist wiederum das Resultat einer Induktion. So stützt sich zum Beispiel der Beweis, daß jedes Stück Roteisen durch Heizung sich ausdehnt, auf die Prämisse, daß jedes Stück Eisen

\footnotetext{
${ }^{1}$ Siehe M. Black, Induction, 1967. Für vollständige bibliographische Angaben siehe die Bibliographie am Ende dieses Beitrags.
} 
sich durch Heizung ausdehnt, und für diese letzte braucht man wiederum eine allgemeine sichernde Prämisse.

Man kann den Begriff ‘Wahrscheinlichkeits einführen, wenn man die Wahrheit der Konklusion im Verhältnis zur Zahl der wahrgenommenen Einzelfälle schätzt und eine Voraussage macht. Die wahrscheinliche Induktion führt aufgrund einer relativen Häufigkeit zu einer Konklusion.

Dies sind sehr einfache Erwägungen zum Problem der Induktion, die lediglich das Thema dieses Beitrags erhellen sollen. Ich hoffe, es ist deutlich geworden, daß das Induktionsverfahren im Gegensatz zu dem logisch kräftigeren Verfahren der Deduktion nicht ohne Schwierigkeiten ist und daß diese Schwierigkeiten für die Verfassung einer Wissenschaft folgenreich sind: Die Axiome einer Wissenschaft sind nicht immer absolut sicher.

Im klassischen Altertum wurde der Induktion bereits eine bedeutende Rolle eingeräumt. Plato hatte ein sehr strenges Ideal der menschlichen Erkenntnis entworfen: Alle Erkenntnis soll auf einen festen Gegenstand, die Platonische Idee, bezogen sein. Obgleich Plato den konkreten materiellen Gegenständen ein gewisse Art von Sein zuschrieb, spielt die Erkenntnis dieser materiellen Objekte für ihn kaum eine Rolle auf dem Weg zur wahren Erkenntnis. Sie ist nur Anlaß dazu.

Aristoteles war anderer Meinung. Er hat als erster Elemente einer Theorie der Induktion formuliert. Obgleich Aristoteles manchmal als \&Vater der Induktionstheorie bezeichnet wird, hat er nicht systematisch im Rahmen eines Traktates über den Begriff der Induktion geschrieben.

Im vorliegenden Beitrag soll die Theorie der Induktion dargelegt werden, die man in einer Quaestio eines anonymen mittelalterlichen Kommentars zu den Analytica Priora findet, der einst zu Unrecht Johannes Duns Scotus (1266-1308/9) zugeschrieben wurde; es handelt sich also um eine Quaestio des Pseudo-Johannes Duns Scotus. In dieser Quaestio findet man die meinens Erachtens bemerkenswerte Theorie, daß die Prinzipien der Naturwissenschaft nur wahrscheinlich sind, und zwar der Unmöglichkeit wegen, unter Berücksichtigung aller Fälle zu induzieren. Das quantitative Problem der Induktion wird hier also formuliert. Ich werde versuchen, diese Auffassung mit anderen (älteren und jüngeren) mittelalterlichen Auffassungen zu kontrastieren und sie historisch einzuordnen.

Um dieses Thema zu verdeutlichen, werde ich erstens (§ 2) die Elemente der Induktionstheorie des Aristoteles aufgrund seiner logischen 
Schriften darstellen. Ich werde in diesem Beitrag nicht eine bestimmte Interpretation dieser Theorie verteidigen, sondern nur die Elemente kurz andeuten, die von Pseudo-Duns Scotus und einigen anderen mittelalterlichen Philosophen benutzt wurden. Weiter ( $\$ 3$ ) werde ich kurz, nur um die Theorie des Pseudo-Duns Scotus zu situieren, die Auffassungen Alberts des Großen (1200-1280) und des Thomas von Aquin (1224-1275) besprechen, insofern diese sich auf das Thema beziehen. Dann (\$4) folgt der Text unserer Quaestio: Einleitung (\$ 4.1), Übersetzung (§4.2), Analyse und Kommentar (§ 4.3). Schließlich (§ 5) werde ich kurz die Hauptlinien der Auffassungen des Johannes Buridanus (1300-kurz nach 1358) und des Marsilius von Inghen (kurz vor 1340-1396) besprechen, weil diese meines Erachtens der Theorie des Pseudo-Duns Scotus ähneln.

\section{§2. Aristoteles}

Manchmal benutzt Aristoteles das Wort (Induktion in einem weiteren Sinne, so zum Beispiel in Physica I, ii, 185 a 12-14, wo er sagt, daß cwir als Ausgangsgrundsatz annehmen sollen, daß die Naturdinge, entweder alle oder zum Teil, sich bewegen oder verändern; dies ist klar aufgrund von Induktions. Aristoteles meint offensichtlich, daß dies klar ist aufgrund der methodischen Erfahrung von mehreren Einzelfällen sich bewegender und verändernder Naturgebilde.

In seinen logischen Schriften gibt Aristoteles Elemente einer Theorie der Induktion an:

1) in seinen Analytica Priora II, xxi, erklärt er, auf welche Weise Unwissenheit einer Konklusion vereinbar ist mit der Kenntnis der Prämissen. Man soll unterscheiden zwischen dem Wissen des Allgemeinen und dem Wissen des Einzelnen (67 a 19-21). ‘Dies gilt auch', sagt Aristoteles (67 a 21-26), (für die Theorie in Platos Meno, daß Lernen Wiedererinnerung ist. Denn in keinem Fall trifft es zu, daß wir im voraus etwas Einzelnes wissen, sondern zugleich mit der Induktion gewinnen wir die Erkenntnis des Einzelnen, insofern wir uns an es wiedererinnern. Denn manches wissen wir unmittelbar, zum Beispiel daß etwas eine Winkelsumme von zwei rechten hat, wenn wir wissen daß es ein Dreieck ist. So verhält es sich auch in anderen Fällen. 
Man könnte hier den Schluß ziehen, daß die Theorie der Induktion für Aristoteles der Ersatz ist für Platos Theorie der Anamnesis ${ }^{2}$. Aristoteles' Induktionstheorie scheint mir jedoch eine Kritik dieser Theorie zu sein: Er legt den logischen Primat in die Erkenntnis des aktuell Existierenden. Aristoteles illustriert seine Theorie mit einem Beispiel: Aufgrund der Erfahrung nur eines Dinges wird das Universelle erfaßt ${ }^{3}$ - was sich versteht für die Mathematik. Aristoteles schließt nicht auf aktuelle Vorkenntnis, sondern implizites (potentielles) Wissen ist gefordert.

2. In Topice I, xii sagt Aristoteles, daß es zwei Arten dialektischer Argumentation gibt: Induktion und Deduktion. Unter Deduktion versteht Aristoteles einen Syllogismus, d.h. eine logisch notwendige Ableitung einer Konklusion aus Prämissen. Induktion ist das Fortschreiten von individuellen Dingen zum Universellen. Aristoteles gibt ein Beispiel: «Wenn der sachverständige Steuermann der beste Steuermann ist und wenn der sachverständige Wagenlenker der beste Wagenlenker ist, ist im allgemeinen der Sachverständige der Beste in jedem individuellen Fall. Induktion ist überzeugender, deutlicher und bekannter für die sinnliche Erfahrung, und sie ist gemeinschaftlicher Besitz für die meisten Leute; Syllogismus aber ist kräftiger und effektiver gegen Diskussionsgegner. In diesem induktiven Argument geht Aristoteles von der Erkenntnis einiger bestimmter Einzelfälle aus, um zu einer allgemeinen Konklusion zu gelangen.

3) In Analytica Priora II, xxiii sagt Aristoteles, daß alle unsere Überzeugungen entweder durch Syllogismus oder durch Induktion zustande kommen (68 b 13-14). Er fährt fort: Induktion, oder induktive Argumentation, besteht darin, daß man mittels des einen Außenbegriffs schließt, $\mathrm{da} B$ der andere dem Mittelbegriff zukommt. Zum Beispiel beweist man, wenn B der Mittelbegriff von A und C ist, mittels C, daß A dem B zukommt. Auf diese Weise machen wir Induktionen. Es stehe zum Beispiel (A) für das, was lange lebt, (B) für das, was keine Galle besitzt, und \& $C$, für die einzelnen (Arten), die lange leben, wie z. B. Mensch, Pferd und Maulesel, dann kommt $A$ dem $C$ als ganzem zu (denn jedes $C$ ist langlebig) (68 b 15-22), 4 .

\footnotetext{
2 Siehe auch Analytica Posteriora II, xix, 99 b 27-32.

3 Ibidem.

${ }^{4}$ Nach H. Tredennick (Fußnote $b$ auf S. 514 der Loeb-Ausgabe (1938)) soll dieser eingeklammerte Satz gestrichen werden, weil er eine Petitio principii enthält und irrelevant ist. W.D. Ross (Aristotle's Prior and Posterior Analytics, 1949, S. 468) glaubt
} 
Diese Textstelle wirft ein besonders schwieriges Interpretationsproblem auf ${ }^{5}$. Es genügt hier zu bemerken, daß Aristoteles dort Induktion auf Deduktion reduziert und somit als logisch gültige Argumentation präsentiert. Dabei beachte man, daß Aristoteles hier als induzierte Fälle Arten nennt und nicht Individuen. Da nach Aristoteles die Zahl der Arten in der Welt begrenzt ist - während die Zahl der individuellen Dinge unbegrenzt ist ${ }^{6}-$, ,önnte er auf diese Weise Induktion als 'geschlossen' präsentieren und auf einen Syllogismus reduzieren.

4) In den Analytica Priora II, xxiv bespricht Aristoteles die Argumentationsform des 'Beispiels?. Aristoteles ist nicht besonders klar in seiner Darlegung ${ }^{7}$. Ein Beispiel verdeutlicht, was er meint: Wenn man beweisen will, daß ein Krieg der Athener gegen Theben schlecht ist, soll man zuerst wissen, daß Krieg gegen Nachbarn schlecht ist. Man kann das glauben aufgrund gleichartiger Fälle, zum Beispiel aufgrund dessen, daß der Krieg der Thebaner gegen Phokis schlecht ist. Man kann jetzt schließen, daß Krieg gegen Theben schlecht ist.

Die Argumentationsform des Beispiels ist nach Aristoteles also eine Argumentation, die von einem individuellen Fall (eine Eigenschaft gehört einem individuellen Ding zu) ausgeht und zu einem anderen individuellen Fall hinführt. Das Beispiel gleicht teilweise der Induktion, aber der Unterschied zur Induktion ist, daß man nicht von Einzelfällen auf ein Allgemeines hin argumentiert, sondern bei einem anderen eine Meinung über etwas verursacht. Das Beispiel führt daher nicht zu wissenschaftlicher Erkenntnis.

5) In dem berühmten Kapitel II, xix der Analytica Posteriora (‘Wissenschaftslehre des Aristoteles) ) erörtert Aristoteles die verschiedenen Arten der Erkenntnis, von der sinnlichen Erfahrung an bis hin zum intellektuellen Erfassen der ersten Prinzipien durch den Geist ${ }^{8}$. Auf diese Weise hat

zwar, Tredennick habe recht, hat aber Bedenken, diesen Satz zu streichen, weil er in allen Handschriften überliefert sei.

${ }^{5}$ Siehe zum Beispiel: K. von Fritz, Die Epagoge bei Aristoteles, München 1964; W. Hess, (Erfahrung und Intuition bei Aristoteles), 1970; N. Tsouyopoulos, (Die induktive Methode und das Induktionsproblem in der griechischen Philosophie?, 1974.

${ }^{6}$ Siehe Aristoteles, Rhetorica, A, II, 1356 b 31; Metaphysica A, II, 994 b 22; B, IV, 999 a 27; Analytica Posteriora I, xxiv, 86 a 6.

${ }_{7}^{7}$ Siehe Ross, Aristotle's Prior and Posterior Analytics, 1949, S. 488.

${ }^{8}$ Siehe auch unten $\S 5$. 
der Mensch wissenschaftliche Erkenntnis, welche immer wahr ist und sich nicht irren kann $(100$ b $5 ; 10)$. Dieser Weg, so Aristoteles, ist Induktion.

Die soeben genannten Textstellen sind nicht einfach logisch miteinander in Einklang zu bringen. Ich kann hier, wie bereits oben gesagt, nicht die umfangreiche Literatur besprechen. Es mag genügen, diejenigen Aspekte der Aristotelischen Induktionstheorie zu erwähnen, die in der zu besprechenden mittelalterlichen Quaestio eine Rolle spielen. Mir scheint es, wie ebenfalls oben gesagt, daß bei Aristoteles Induktion ein ziemlich einfacher Ersatz der Platonischen Anamnesistheorie ist und daß er nicht alle Probleme der unvollständigen - aber wissenschaftlich fruchtbaren und der vollständigen Induktion gesehen hat. Soweit ich sehe, sagt Aristoteles nicht explizit, daß die Naturwissenschaft des quantitativen Problems der Induktion wegen weniger sicher ist und nur wahrscheinliche Ausgangsprinzipien hat.

Dennoch hat Aristoteles an anderen Textstellen auch hinsichtlich der Genauigkeit der Beweismethode der Wissenschaften Unterschiede gemacht. In Metaphysik XI, vi (1063 b 36-1064 a 7) ${ }^{9}$ heißt es, daß die Metaphysik ihren Ausgangspunkt hat im Seienden als Seienden und daß die anderen Wissenschaften, zum Beispiel eine der Naturwissenschaften, eine bestimmte Klasse der Dinge markiert und die Dinge nicht als Seiende, sondern als spezielle Seiende studiert. Meines Erachtens meint Aristoteles, daß die Naturwissenschaft aus diesem Grund auch weniger sicher ist.

In Metaphysik VI, I (1025 b 7-11) lehrt Aristoteles, daß cim Allgemeinen jedes diskursive Wissen oder Wissen, das an einem Diskurs Anteil hat, sich mit Ursachen und Prinzipien beschäftigt, die entweder mehr <oder weniger> genau oder einfacher sind s. Aristoteles fügt hinzu (Z. 11-14), $\mathrm{da} B$ die verschiedenen Wissenschaften auf diese Weise notwendiger oder schwächer beweisen.

Man könnte sagen, daß Naturwissenschaft in dieser Hinsicht weniger genau ist der Prinzipien wegen, weil diese Prinzipien im Vergleich mit denen der Metaphysik zusammengesetzt sind. Man könnte also sagen, daß die geringere Genauigkeit der Naturwissenschaft nicht eine Folge der beschränkten Zahl der erfahrenen Einzelfälle ist. Aristoteles scheint dem-

${ }^{9}$ Vergleiche Metaphysica XI, VII. Siehe auch Analytica Posteriora I, xxvii; Metaphysica A, II, 982 a 25-28; ibidem M, III, 1078 a 9-17. 
nach das am Anfang dieses Beitrags genannte quantitative Problem der Induktion nicht zu sehen.

\section{§3. Albert der Große (ca. 1200-1280) und Thomas von Aquin (1224-1275)}

Ich lasse nun einige Bemerkungen über die Induktionstheorien Alberts des Großen und Thomas von Aquins folgen, um der Theorie des PseudoJohannes Duns Scotus einen Hintergrund zu geben.

Albert der Große unterscheidet in seinem Kommentar zu Aristoteles' Analytica Priora zwischen einer vollständigen und einer wahrscheinlichen Induktion. Für eine vollständige Induktion soll man in summa (‘vollständigs) induzieren, während man nicht alle Einzelfälle Stück für Stück aufzählt, sondern zusammenfaßt mit $e t$ sic de singulis (sund so weiter) 10; dies ist nicht nur gültig für einen Dialecticus, sondern auch für einen Demonstrator. Eine wahrscheinliche Induktion induziert über viele, aber nicht alle Einzelfälle und führt zu einer Konklusion, vorausgesetzt, daß es kein Gegenbeispiel gibt, sagt Albert ${ }^{11}$. Diese Induktion ist nicht gültig in der Wissenschaft oder der beweiskräftigen Erkenntnis: Sie soll in der Topik diskutiert werden, sagt er. Er bringt augenscheinlich die offene Induktion nicht in Verbindung mit Wissenschaft und kann in seiner Theorie auf diese Weise, wie unten ${ }^{12}$ deutlicher wird, mit Pseudo-Duns Scotus kontrastiert werden.

Thomas von Aquin folgt den Grundgedanken seines Lehrers Albertus Magnus; er unterscheidet auch zwischen vollständiger und unvollständiger Induktion. Unyollständige Induktion findet sich zum Beispiel in der Rhetorik, also im dialektischen, nicht im beweiskräftigen Denken, weil die Objekte dieses Denkens nicht stabil sind. Eine Art unvollständiger Induktion ist die Argumentationsform des Beispiels ${ }^{13}$. Vollständige Induktion führt zu universeller Erkenntnis und ist die Art von Induktion, die zur Wissenschaft führt.

${ }^{10}$ Siehe auch A. Mansion, d'Induction chez Albert le Grand, 1906.

${ }^{11}$ Kommentar zu den Analytica Priora, Liber II, Tractatus vii, caput iv in B. Alberti Magni Opera omnia (...) Teil I, 1890, p. 795a.

12 Siehe unten, \$ 4.

${ }^{13}$ Siehe oben, $\S 2$. 


\section{E.P. Bos}

Thomas interpretiert das Universelle als eine Art ewiger Eigenschaft. In der Mathematik sind das zum Beispiel Gestalt und Zahl als Eigenschaften aller mathematischen Objekte. Der Geist ist imstande, kraft seiner Hinneigung zum Wahren das Universelle zu erfassen, sagt Tho$\operatorname{mas}^{14}$.

Thomas unterscheidet auch nach dem Grad der Gewißheit der Wissenschaften. In seinem Kommentar (Quaestiones) zur Schrift De Trinitate des Boethius diskutiert er die Methoden der theoretischen Wissenschaften. Die Mathematik ist die sicherste Wissenschaft weil sie die formellen inneren Ursachen untersucht; sie ist sicherer als die Metaphysik, insofern diese für uns Menschen zu hoch ist. Die Naturwissenschaft ist weniger gewiß, weil sie nur die äußeren Ursachen, d.h. Wirk- und Zielursache, untersucht ${ }^{15}$.

Man könnte feststellen, glaube ich, daß der Grad der Gewißheit einer Wissenschaft hier vom Standpunkt der menschlichen Erkenntnis aus bestimmt wird: Naturwissenschaft ist in dieser Hinsicht dem Menschen proportional. Weil aber die Dinge, mit denen der Mensch primär bekannt ist, nämlich die Naturdinge, veränderlich sind und die menschliche Vernunft primär die Außenseite der Dinge kennt ${ }^{16}$, ist die naturwissenschaftliche Erkenntnis weniger genau als die mathematische Erkenntnis. Es sind nicht quantitative Aspekte, die den Grad der Gewißheit bestimmen, sondern, wie ich sie hier nenne, (qualitative) Aspekte, d.h. Aspekte der Betrachtung der Dinge.

\section{§4. Pseudo-Johannes Duns Scotus über Induktion}

In der Edition Vivès (1891-1895) der Opera omnia Johannis Duns Scoti finden sich viele Kommentare und Traktate, die lange Zeit zu Unrecht von den Editoren dem Doctor subtilis zugeschrieben wurden ${ }^{17}$. Die unechten Werke konnten teilweise bestimmten Autoren attribuiert werden: Tho-

\footnotetext{
14 Thomas von Aquin, Expositio in Analytica Posteriora, Liber II, lectio xx, nr. 593.

15 Siehe A.A. Maurer, St. Thomas Aquinas, The Division and Methods of the Sciences, 1963, S. xxxiii-xxoxiv.

16 Siehe Thomas von Aquin, Summa theologiae, I-II, qu. 91, art. 4, in corpore.

${ }^{17}$ C. Balić gibt eine knappe Übersicht in The Life and Works of John Duns Scotus, 1965.
} 
mas von Erfurt (fl. um 1300), Vitalis de Furno (ca. 1260-1327), Antonius Andreas (14. Jahrhundert) - alles Meister des 14. Jahrhunderts.

Auch der Kommentar (Quaestiones) zu den Analytica Priora ist zu Unrecht Duns Scotus zugeschrieben worden, wie C. Balić in einer Übersicht erwähnt ${ }^{18}$. Er selber gibt dort keine Argumente. Ch. Lohr ${ }^{19}$ nennt in seinem Verzeichnis der mittelalterlichen Aristoteleskommentare keine Handschriften dieses Kommentars. Nach Lohr sind die Quaestiones super libros Priorum in neun verschiedenen Editionen überliefert: Venedig 1504, 1512; Pavia 1520; Venedig 1586, 1600, 1610; Ursellis 1622; Lyon (Edition Wadding) 1639; Paris (Edition Vivès) 1891.

Einige Gelehrte haben den Kommentar zu den Analytica Priora mit demjenigen zu den Analytica Posteriora in Verbindung gebracht. Aus Ch. Lohrs Übersicht erfahren wir, daß eine der zwei Handschriften, nämlich Oxford, Magdalen College 162 (15. Jahrhundert), f. 183-(250) ${ }^{20}$ folgendes Explizit hat: Expliciunt quaestiones et tituli tam primi libri quam secundi Posteriorum Analyticorum datae a domino Johanne de Sancto Germano de Cornubia ('Explizit der Quaestiones und Titel des ersten und zweiten Buches der Analytica Posteriora, ediert (?) von Herrn Johannes von SaintGermain von Cornwall), (Dataes meint vielleicht nur cediert und nicht 'verfaßst. Aus der anderen Handschrift (Toledo, Biblioteca del Cabildo, 19.25 (14. Jahrhundert)) zitiert Lohr kein Explizit. Dieser Kommentar zu den Analytica Posteriora ist auch in den oben genannten gedruckten Editionen enthalten.

Nach M.J. Grajewski ${ }^{21}$ sind die beiden Kommentare sprobably the work of John of Cornubia) (Cornubia ist Cornwall). Er erörtert diese Vermutung nicht näher. E. Gilson ${ }^{22}$ sagt, daß U. Smeets ${ }^{23}$ die Kommentare Johannes de Cornubia zuschreibt. Mir war diese maschinenschriftliche Arbeit leider nicht zugänglich. Auch konnte ich die Arbeit von B. Goelz über die echten und unechten Werken des Duns Scotus nicht einse-

\footnotetext{
${ }^{18}$ Siehe Anm. 17.

${ }^{19}$ Siehe Ch. Lohr, Medieval Latin Aristotle Commentaries), 1970, S. 194.

${ }^{20}$ Die Klammern sind nach Lohr, siehe Anm. 19.

${ }^{21}$ Siehe M.J. Grajewski, ,Duns Scotus in the Light of Modern Research, 1942, S. 180.

${ }^{22}$ Siehe E. Gilson, Jean Duns Scot. Introduction à ses positions fondamentales, 1952, S. 180.

${ }^{23}$ U. Smeets, Lineamenta bibliographiae Scotisticae, 1942, S. 8-9.
} 
hen ${ }^{24}$. Es gibt meines Erachtens keinen Anlaß zu der Annahme, daß die beiden Kommentare nur einem Verfasser zugehören.

In den modernen Studien von S. Read ${ }^{25}$, G. Priest und R. Routley ${ }^{26}, \mathrm{P}$. Thom $^{27}$, B. Mates ${ }^{28}$ und A.Ch.S. McDermott ${ }^{29}$ über logische Aspekte des Kommentars zu den Analytica Priora wird das Problem der Datierung und Urheberschaft nicht besprochen. Ich bin jetzt nicht imstande dieses Problem zu lösen. Ich werde zu dem Schluß kommen (\$ 6), daß die Theorie der Induktion, die in unserem Kommentar vertreten wird, sich von derjenigen unterscheidet, die sich in den unbezweifelbar echten Werken des Johannes Duns Scotus findet. Aufgrund dieses Unterschieds kann die Theorie in unserem Kommentar nicht dem Doctor Subtilis zugeschrieben werden. Ferner ähnelt die Induktionstheorie des Pseudo-Duns Scotus sehr der Auffassung von Johannes Buridanus (1300-kurz nach 1358) und von Marsilius von Inghen (kurz vor 1340-1396). Daher gehört unser Kommentar vielleicht in die erste Hälfte des 14. Jahrhunderts, wie andere unechte Kommentare in der Edition Vivès.

\section{$\S 4.1$ Text}

Pseudo-Johannes Duns Scotus, Super librum secundum Priorum Analyticorum, quaestio VIII, ed. Paris (Vivès), 1891, S. 195a-197b (zweispaltig)

N.B.: ed.: Edition Vivès

$<\ldots\rangle$ : Hinzufügung des Herausgebers hier

${ }^{24}$ Siehe B. Goelz, <Die echten und unechten Werke des Duns Skotus nach dem gegenwärtigen Stand der Forschung', 1934.

25 Siehe S. Read, Self-Reference and Validity, 1979.

${ }^{26}$ G. Priest und R. Routley, 'Lessons from Pseudo-Scotus», 1982.

${ }^{27}$ P. Thom, Conversion of Propositions Containing Singular or Quantified Terms in Pseudo-Scotus', 1982.

28 B. Mates, ‘Pseudo Scotus on the Soundness of Consequentiae;, 1965.

29 A.Ch.S. McDermott, Notes on the Assertoric and Modal Propositional Logic of the Pseudo-Scotus', 1972. 
[QUAESTIO VIII]

Utrum ad bonam inductionem oportet inducere in omnibus singularibus ${ }^{\text {a }}$

[Rationes quod non]

Arguitur quod non:

(I.1) Singularia sunt infinita, ut dicit Porphyrius ${ }^{\text {b }}$; sed infinita non possunt pertransiri; igitur non potest fieri inductio in omnibus singularibus.

(I.2) Secundo: vel induceretur in omnibus sub propria forma quodlibet enumerando singulatim, aut in omnibus sub clausula communi, ut dicendo et sic de singulis, aut in aliquibus sub propria forma, et in aliquibus sub clausula communi. Non primo modo propter infinitatem singularium, ut dictum est ${ }^{c}$; nec secundo modo: quia tunc inducere non esset aliud quam ponere propositionem universalem; nec tertio modo: quia omnes singulares sunt eiusdem rationis; igitur qua ratione debet induci in aliquibus sub propria forma, eadem ratione in omnibus.

(I.3) Tertio: in nulla argumentatione dialectica debet committi petitio principii; sed inducendo in aliquibus singularibus sub propria forma, et in aliis sub clausula communi, fit petitio principii; igitur et cetera. Maior patet: quia argumentatio dialectica semper probat conclusionem, et ubi est petitio principii, nulla est probatio. Minor tenet: quia aeque nota est universalis quae debet induci sicut clausula communis per quam inducitur.

(I.4) Quarto: quia illud sufficit ad bonam inductionem quo posito salvatur definitio inductionis; sed posito quod fiat inductio in aliquibus singularibus et non in aliis (p. 195b) salvaturd definitio inductionis; igitur et cetera. Maior nota est, et minor probatur: quia inductio est progressio et cetera.

\footnotetext{
a Ich habe stillschweigend die Interpunktion und Orthographie des lateinischen Textes an einigen Stellen etwas normalisiert (zum Beispiel: probatur: quias statt probatur, quia), (numquam statt (nunquam) etc.); ich habe einige Wörter kursiviert, den Text mit Nummern versehen, Kommata und Großbuchstaben eingeführt. Diese Änderungen berühren keineswegs die Interpretation des Textes.

b Porphyrius, Isagoge, ed. A. Busse, in Commentaria in Aristotelem Graeca, Teil IV (1), 1887, S. 6, Z. 17. in Porphyrii Isagoge, Translatio Boethii, ed. L. Minio-Paluello, 1966, S. 12, Z. $12-13$.

¿ Siehe oben, den Text, $\S$ I.1.

d salvatur] ego; non salvatur ed.
} 
Modo ista definitio salvatur posito quod non fiat inductio nisi in duobus singularibus.

[Oppositum]

\section{Oppositum arguitur:}

(II.1) quia illud sequitur ad bonitatem inductionis quod sequitur ad veritatem universalis inductae; sed ad veritatem universalis inductae sequitur veritas cuiuslibet eius singularis; igitur et cetera.

(II.2) Secundo: quia inductio idem est quod locus a partibus totius in quantitate ad suum totum ${ }^{\mathrm{e}}$; sed iste locus non tenet nisi in omnibus partibus sufficienter enumeratis.

(II.3) Tertio: nisi ad bonam inductionem requireretur inductio in omnibus singularibus, sequeretur quod in bona consequentia antecedens esset verum et consequens falsum, quod est contra definitionem consequentiae datae. Consequentia patet: quia si inducatur solum in aliquibus singularibus respectu alicuius ${ }^{f}$ praedicati, possibile est quod illud praedicatum conveniat singularibus in quibus inducitur et quod non conveniat aliis; igitur universalis inducta quae est consequens, est falsa, et tamen antecedens est verum.

(II.4) Quarto sequeretur quod inductio non posset reduci ad figuram, quia numquam potest esse universalis per quam reducitur nisi enumeratis omnibus singularibus.

(II.5) Quinto per Aristotelem primo Topicorum8, ubi dicit quod, si positio fuerit universalis et fit instantia in uno distributo, est problema.

[Conclusiones]

Ad quaestionem ponuntur conclusiones:

(III.1) Prima est quod inductio non valet ad concludendum de necessitate nisi inducatur in omnibus singularibus. Probatur: quia illa consequentia non est necessaria, in qua ${ }^{\mathrm{h}}$ possibile est antecedens esse verum et conse-

\footnotetext{
' Petrus Hispanus, Tractatus, ed. L.M. de Rijk, S. 64, Z. 24-S. 65, Z. 30. Man ziehe zu Rate: N.J. Green-Pedersen, The Tradition of the Topics in the Middle Ages, 1984, S. 48.

$\mathrm{f}$ alicuius] ego; alterius $\mathrm{ed}$.

8 Aristoteles, Topica, I, xi, 104 b 32-35 (wahrscheinlich).

h In qua] ego; et quia ed.
} 
quens falsum. Sedi sic est in inductione, si non inducatur in omnibus singularibus, ut probat tertia ratio post oppositum; igitur et cetera.

(III.2) (p. 196a) Secunda conclusio quod inductio non valet ad concludendum evidenter - supposito quod inducatur in omnibus singularibus - nisi coassumatur propositio universalis mediante qua ex singularibus fit syllogismus. Verbi gratia: posito quod non essent nisi tres homines, scilicet Socrates et Plato et Cicero, tunc sequitur necessario Socrates currit et Plato currit et Cicero currit, igitur omnis homo currit; tamen non sequitur evidenter nisi addatur ista universalis omnis homo est Socrates et Plato et Cicero qua apposita est consequentia evidens.

(III.3) Tertia conclusio est quod ad habendum opinionem probabilem, fidem vel persuasionem de conclusione universali, sufficit inducere in aliquibus singularibus, licet non inducatur in omnibus. Et ideo multae inductiones sunt bonae arguendo absolute absque hoc quod in omnibus singularibus inducatur.

(III.3.1) Probatur: quia fortius potest movere inductio in aliquibus singularibus ad faciendum probabilitatem, fidem vel opinionem de propositione universali quam potest solum exemplum per unum singulare movere ad faciendum fidem de alio singulari; sed certum est quod solum exemplum per unum singulare facit opinionem de alio singulari; igitur multo fortius inductio in aliquibus singularibus facit opinionem de universali.

(III.3.2) Secundo: illud sufficit ad bonam inductionem quod sufficit ad hoc quod universalis inducta sit probabilis; sed ad probabilitatem universalis inductae sufficit inductio in aliquibus singularibus, licet non in omnibus, igitur et cetera.

Maior probatur: quia finis argumentationis dialecticae non est demonstrare conclusionem evidenter, sed est facere probabilitatem et opinionem de conclusione.

Et minor patet: quia, si fiat inductio in aliquibus singularibus et non habetur evidentia, ut ratio, quoniam ita fit in aliis, oportet quod respondens concedat universalem inductam, vel quod det instantiam in aliquo singulari, vel quod assignet differentiam quare non est ita de aliis singularibus sicut de istis, vel erit reductus ad metam inopinabilem. (III.3.3) Tertio: quia multa principia naturalia fiunt nobis evidentia propter sensum, memoriam et experientiam, ut ista ommis ignis est calidus;

\footnotetext{
iSed] ego; et $e d$.

i Siehe oben, in dem lateinischen Text, § II.3.
} 
omne grave existens sursum non impeditum naturaliter descendit deorsum et consimilia. Quae facta sunt evidentia per inductionem, et non in omnibus singularibus, ut notum est; igitur sumitur in aliquibus singularibus, et non in omnibus.

Et si quaeratur: (in quot singularibus oportet inducere?», respondetur quod de illis non potest assignari certus numerus. Sed quandoque oportet inducere in pluribus, quandoque in paucioribus secundum diversitatem materiae, et secundum diversitatem intellectionis intellectus qui debet assentire universali.

(III.4) Quarta conclusio: quod in aliquibus, ut respectu praedicatorum per accidens, non sufficit inducere in aliquibus singularibus, sed oportet inducere in omnibus. Patet: quia in aliquibus est materia talis quod ad habendum opinionem vel probabilitatem de universali non sufficit quod inducatur in aliquibus singularibus, sed oportet quod inducatur in omnibus.

(III.5) Ex praedictis sequitur quod intellectus quodammodo magis libere assentit quibusdam quam sensus faciat, sicut in naturalibus propter evidentiam quam habet de aliquibus singularibus statim assentit ita est de omnibus. Et hoc est, quia in illa materia non potest melius <in omnibus> quam in aliquibus, ut in mathematicis non sufficeret intellectus.

[Ad rationes] [Ad rationes ante oppositum]

(IV.1.1) Ad primam dico quod non oportet inducere in omnibus, et si sit tale praedicatum quod oportet inducere in omnibus, tunc <oportet> in aliquibus inducere in propria forma, scilicet aliquas singulares sumendo, et in aliis sub clausula communi dicendo et sic de singulis.

(IV.1.2) Ad secundam dico quod non omnes sunt eiusdem rationis: quia aliquae sunt notae (f. 197a) per sensum, et in talibus debet induci sub propria forma, et in aliis sub clausula communi.

(IV.1.3; IV.1.4) Ad tertiam et ad quartam simul dicitur, quod in inductione non committitur petitio principii: quia ex quo aliquae singulares sunt notae et non apparet instantia in aliis, ut dicatur ${ }^{k}$, quare non debet ita esse de aliis, concludenda est universalis.

k dicaturl ego; dicitur ed. 
Ad ultimum quod ad habendum opinionem de conclusione sufficit inducere (p. 197b) in aliquibus singularibus. Sed ad inferendum de necessitate oportet inducere in omnibus. Ideo in aliquibus debet suppleri quod inductio est progressio ab aliquibus singularibus vel ab omnibus sufficienter enumeratis ad conclusionem universalem.

$$
\text { [Ad rationes post oppositum] }
$$

Rationes post oppositum probant quod non valet inductio ad concludendum de necessitate vel evidenter nisi inducatur in omnibus singularibus. Et sic sit dictum ad quaesitum felici exitu.

\section{\$4.2 Übersetzung}

Pseudo-Johannes Duns Scotus, Kommentar (Quaestiones) zum zweiten Buch der Analytica Priora, Quaestio viii.

N.B.: <...> heißt: Verdeutlichung des Übersetzers.

\section{[8. PROBLEM]}

Ist es notwendig, für eine gültige Induktion über alle einzelne Fälle zu induzieren?

[Argumente, daß dies nicht nötig sei]

Man argumentiert, daß das nicht nötig sei:

(L.1) Einzelne Fälle sind unendlich <der Zahl nach>, wie Porphyrius sagt; aber <eine> unendliche <Zahl von> Fällen kann man nicht durchlaufen; also kann es keine Induktion über alle einzelne Fälle geben.

(I.2) Zum zweiten: Entweder (1) würde sich die Induktion über alle Fälle unter deren eigener Form vollziehen, wobei jedes einzelne Stück für Stück aufgezählt würde; oder (2) sie würde sich über alle Fälle unter einem allgemeinen Ausdruck vollziehen, nämlich sund so bei allen Fällen;; oder (3) sie würde sich über einige Fälle unter der eigenen Form und über andere Fälle unter einem allgemeinen Ausdruck vollziehen. In der ersten Weise (1) vollzieht sie sich nicht wegen der unendlichen Zahl der einzelnen Fälle, wie bereits gesagt wurde; auch nicht in der zweiten Weise (2), 
weil sonst induzieren nichts anderes wäre als eine universelle Aussage vorbringen; auch nicht in der dritten Weise (3), weil alle einzelnen Fälle von derselben Art sind; deshalb muß man aus demselben Grund, aus dem man in einigen Fällen unter deren eigener Form induzieren muß, dies in allen Fällen tun.

(I.3) Zum dritten: In keiner dialektischen Argumentation darf man den Trugschluß der Petitio principii ( $T$ Trugschluß der Annahme von etwas zu Beweisendem') begehen; aber wenn man in einer Zahl einzelner Fälle unter deren eigener Form und in anderen Fälle unter einem allgemeinen Ausdruck induziert, findet eine Petitio principii statt; deshalb (...) und so weiter. Die obere Prämisse ist deutlich: Eine dialektische Argumentation beweist immer eine Konklusion, und im Falle einer Petitio principii gibt es keinen Beweis. Die andere Prämisse ist wahr: Die universelle Aussage, die man induzieren soll, ist ebensogut bekannt wie der allgemeine Ausdruck, mit dessen Hilfe man induziert.

(I.4) Zum vierten: Für eine gültige Induktion genügt dasjenige, bei dessen Gegebensein der Definition der Induktion entsprochen wird. Aber falls die Induktion sich in einer bestimmten Zahl einzelner Fälle vollzieht und in anderen Fällen nicht, wird der Definition der Induktion entsprochen; deshalb (...) und so weiter. Die obere Prämisse ist bekannt; die untere Prämisse wird bewiesen, weil Induktion das Fortschreiten ist (...) und so weiter. Dieser Definition wird bereits dann entsprochen, wenn die Induktion sich nur über zwei einzelne Fälle vollzieht.

[Gegenargumente]

Für das Gegenteil spricht, (II.1) daß aus der Gültigkeit einer Induktion folgt, was aus der Wahrheit der induzierten universellen Aussage folgt; aber aus der Wahrheit einer induzierten universellen Aussage folgt die Wahrheit jeder singulären Aussage, die zu ihr gehört; deshalb (...) und so weiter.

(II.2) Zum zweiten: Eine Induktion ist dasselbe wie der Topos von Teilen eines quantitativen Ganzen zu ihrem Ganzen; aber dieser Topos ist nur gültig, wenn alle Teilen genügend aufgezählt sind.

(II.3) Zum dritten: Wenn nicht für eine gültige Induktion gefordert wäre, $\mathrm{da}$ über alle einzelne Fälle induziert wird, würde folgen, daß in einer gültigen Folgerung der Vordersatz wahr und der Nachsatz falsch wären; dies steht im Widerspruch zu der Definition der gegebenen Folge- 
rung. Die Folgerung ist deutlich: Wenn nur in einigen einzelnen Fällen bezüglich eines Prädikats induziert wird, ist es möglich, daß jenes Prädikat den einzelnen Fällen, über die induziert wird, zukommt und den anderen nicht; folglich ist die induzierte universelle Aussage, die den Nachsatz bildet, falsch, und dennoch ist der Vordersatz wahr.

(II.4) Zum vierten: Es würde folgen, daß es nicht möglich wäre, die Induktion auf eine Figur zu reduzieren: Denn nur dann kann es eine universelle Aussage geben, mittels deren sie reduziert wird, wenn alle einzelnen Fälle aufgezählt worden sind.

(II.5) Zum fünften: Aufgrund <des Textes von> Aristoteles (Topica I), in welchem er sagt, daß dann, wenn eine universelle Aussage vorgebracht wird und es in einem Einzelfall ein Gegenbeispiel gibt, ein Problem vorliegt.

(III)

[Thesen]

Thesen:

(III.1) Erste These: Eine Induktion taugt nur dann zu einem notwendigen Schluß, wenn über alle einzelne Fälle induziert wird. Beweis: Eine Folgerung ist nicht notwendig, in der es möglich ist, daß der Vordersatz wahr und der Nachsatz falsch sind; dies ist aber der Fall bei der Induktion, falls nicht über alle einzelne Fälle induziert wird, wie das dritte $\mathrm{Ge}$ genargument beweist; deshalb (...) und so weiter.

(III.2) Zweite These: Eine Induktion taugt nur dann zu einem evidenten Schluß - vorausgesetzt, daß über alle einzelne Fälle induziert wird -, wenn eine universelle Aussage herangezogen wird, mittels welcher aus singulären <Aussagen> ein Syllogismus entsteht. Zum Beispiel: Unter der Voraussetzung, daß es nur drei Menschen gibt, nämlich Sokrates, Plato und Cicero, folgt notwendigerweise: Sokrates läuft, Plato läuft, und Cicero läuft, also läuft jeder Mensch. Dennoch folgt dies nur evident, wenn diese universelle Aussage herangezogen wird: Alle Menschen sind Sokrates und Plato und Cicero; wenn diese herangezogen worden ist, ist die Folgerung evident.

(III.3) Dritte These: Um eine wahrscheinliche Meinung, Glauben oder Überzeugung mit Bezug auf eine universelle Konklusion zu haben, genügt es, über einige einzelne Fälle zu induzieren, obgleich nicht über alle Fälle. Deshalb sind viele induktive Argumente gültig, wenn man 
schlechthin argumentiert, ohne daß über alle einzelne Fälle induziert würde.

(III.3.1) Beweis: Die Induktion über einige einzelne Fälle kann < die Vernunft> kräftiger bewegen, um Wahrscheinlichkeit, Glauben oder Meinung bezüglich einer universellen Aussage herzustellen, als ein einziges Beispiel von einem einzelnen Fall dies kann, um Glauben bezüglich eines anderen einzelnen Falles herzustellen. Aber es ist sicher, daß ein einziges Beispiel von einem einzelnen Fall eine Meinung über einen anderen einzelnen Fall herstellt; deshalb stellt Induktion auf noch viel kräftigere Weise in einigen einzelnen Fällen eine Meinung bezüglich einer universellen Aussage her.

(III.3.2) Zum zweiten: Für eine gültige Induktion genügt das, was genügt, damit die induzierte universelle Aussage wahrscheinlich ist. Aber für die Wahrscheinlichkeit einer induzierten universellen Aussage genügt die Induktion über einige, wenn auch nicht über alle, einzelne Fälle, deshalb (...) und so weiter.

Die obere Prämisse wird folgendermaßen bewiesen: Das Ziel einer dialektischen Argumentation besteht nicht darin, eine Konklusion evident zu beweisen, sondern Wahrscheinlichkeit und Meinung über eine Konklusion herzustellen.

Die untere Prämisse ist deutlich: Wenn eine Induktion sich über einige einzelne Fälle vollzieht und es keine Evidenz gibt als Argument dafür, $\mathrm{da}$ es auch in anderen Fällen so geschieht, ist es erforderlich, daß der Respondent die induzierte universelle Aussage annimmt oder daß er ein Gegenbeispiel gibt mit Bezug auf irgend einen einzelnen Fall oder daß er einen Unterschied angibt, weshalb es bei den anderen einzelnen Fällen nicht so ist wie bei jenen. Sonst wird er zu einen Endpunkt geführt, den er nicht verteidigen kann.

(III.3.3) Zum dritten: Viele naturwissenschaftliche Prinzipien werden uns evident aufgrund von Sinneswahrnehmung, Erinnerung und Erfahrung, wie zum Beispiel diese: jedes Feuer ist heiß; alles Schweres das sich oben befindet, bewegt sich, wenn es nicht daran gehindert wird, von Natur aus nach unten und dergleichen. Diese wurden durch Induktion evident und nicht über alle einzelne Fälle, wie bekannt; also wird Induktion über einige einzelne Fälle und nicht über alle vollzogen.

Wenn man fragt: ‘Über wie viele einzelne Fälle muß man induzieren??, so lautet die Antwort, daß man von diesen Fällen keine bestimmte Zahl angeben kann; bald muß man über viele Fälle, bald über wenige induzie- 
ren gemäß der Verschiedenheit der Materie und gemäß der Verschiedenheit des Begriffs, der mit einer universellen Aussage zustimmen soll. (III.4) Die vierte These: In bestimmten Fällen, nämlich mit Bezug auf akzidentelle Prädikate, genügt es nicht, über einige einzelne Fälle zu induzieren, sondern man muß über alle Fälle induzieren. Das ist deutlich: In einigen Fällen ist die Materie derart, daß es, um über eine universelle Aussage zu einer Meinung oder einer Wahrscheinlichkeit zu gelangen, nicht genügt, über einige einzelne Fälle zu induzieren, sondern daß man vielmehr über alle Fälle induzieren muß.

(III.5) Aus dem Gesagten folgt, daß der Intellekt gewissermaßen freier bestimmten Dingen zustimmt, als das Sinnesvermögen dies tut. So stimmt er zum Beispiel in natürlichen Dingen der Evidenz wegen, die er über einige einzelne Fällen hat, unmittelbar zu und sagt: So ist es in allen Fällen?. Der Grund ist, weil er in dieser Materie es nicht besser tun kann in allen Fällen als in einigen Fällen, ... ?

(IV.1) [Auseinandersetzung mit den Argumenten für die eine Seite]

(IV.1.1) Zum ersten: Man muß nicht über alle Fälle induzieren, und falls es sich um ein Prädikat von der Art handelt, daß man über alle Fälle induzieren muß, so muß man in einigen Fällen unter der eigenen Form induzieren, nämlich in der Weise, daß man einige einzelne Fälle hernimmt und in anderen Fällen unter einem allgemeinen Ausdruck sagt: cUnd so in den einzelnen Fällen.

(IV.1.2) Zum zweiten: Ich sage, daß nicht alle Fälle derselben Art sind, weil einige bekannt sind durch die Sinne; und bei solchen muß man unter der eigenen Form induzieren, bei anderen Fällen hingegen unter einem allgemeinen Ausdruck.

(IV.1.3; IV.1.4) Zum dritten und zum vierten Argument wird zugleich geantwortet, daß in der Induktion keine Petitio principit ${ }^{\mathrm{m}}$ begangen wird. Denn aufgrund dessen, daß einige einzelne Fälle bekannt sind und sich kein Gegenbeispiel in anderen Fällen zeigt, gemäß welchem man sagt,

${ }^{1}$ Ich muß gestehen, daß ich diesen Satz nicht verstehe. Siehe auch unten, $\S 4.3$, Analyse der Quaestio.

${ }^{\mathrm{m}}$ Siehe auch oben die Edition, $\$$ I.3. 


\section{E. P. Bos}

weshalb es in anderen Fällen nicht so sein muß, ist eine universelle Aussage zu erschließen.

Zum letzten wird gesagt, daß es, um über eine Konklusion eine Meinung zu bekommen, genügt, über einige einzelne Fälle zu induzieren. Um aber notwendigerweise zu schließen, muß man man über alle Fällen induzieren. Deshalb muß man die Definition in einigen Hinsichten ergänzen: Induktion ist das Fortschreiten von einigen einzelnen Propositionen oder von allen genügend aufgezählten Propositionen zu einer universellen Konklusion.

\section{(IV.2)[Auseinandersetzung mit den Argumenten für die andere Seite]}

Die für die andere Seite angeführten Argumente beweisen, daß eine Induktion nur dann zu einem notwendigen oder einem evidenten Schluß taugt, wenn man über alle einzelne Fälle induziert.

Was zum Problem der Quaestio zu sagen war, sei hiermit glücklich zum Abschluß gebracht.

\section{§4.3. Analyse der Quaestio}

Im Folgenden werde ich die Quaestio Schritt für Schritt analysieren, indem ich einzelne Abschnitte kurz zusammenfasse und einen Kommentar (eingeführt mit 〈Kommentar`) hinzufüge.

Kommentar: Ich habe Inductio bona übersetzt mit 'gültige Induktion, d.h. in dieser Quaestio eine Induktion, die auf einen wahrscheinlichen, nicht auf einen notwendigen Schluß führt.

(Ad I) Die Argumente zugunsten einer verneinenden Antwort auf die Quaestio, d.h. daß es nicht notwendig ist, über alle Einzelfälle zu induzieren:

(Ad I.1) Die Einzelfälle sind unendlich.

Kommentar: Nach der Meinung des Opponenten in der Quaestio ist eine vollständige Induktion unmöglich.

(Ad I.2) Entweder (a) man induziert unter der eigenen Form jedes Einzeldinges, was unmöglich ist, oder (b) man induziert unter dem allgemeinen Ausdruck sund so weiter, was soviel hieße wie: eine universelle Aussage machen. 
Kommentar: Im Fall (a) ist wiederum eine unmögliche Aufgabe gestellt, nämlich über alle Fälle zu induzieren; im Fall (b) ist die Induktion unfruchtbar.

Im Fall (c) induziert man teilweise wie (a), teilweise wie (b); das ist unmöglich, denn Induktion soll sich über alle Einzeldinge auf gleiche Weise vollziehen, d.h. unter derselben Form.

Kommentar zu den Argumenten 1 und 2: Diese Argumente beziehen sich auf die Unmöglichkeit der Induktion der Unendlichkeit wegen.

(Ad I.3) Die Form 'und so weiter impliziert eine Petitio principii: Man soll eben beweisen, daß die allgemeine Regel sich auf alle Einzelfälle bezieht. Eine dialektische Argumentation wie die Induktion beweist eine Konklusion, eine Petitio principii aber ist ein Fehlschluß.

(Ad I.4) Auch bei einer Induktion über zwei Einzelfälle wird der Definition der Induktion entsprochen. Diese Definition lautet: 'Die Induktion ist ein Fortschreiten von einigen Einzelfällen zu einer universellen Konklusion.

Kommentar: Für die Definition siehe IV.1.3 und IV.1.4.

(Ad II) Die Gegenargumente sollen zeigen, daß eine vollständige Induktion gefordert ist:

(Ad II.1) Aus der Gültigkeit einer Induktion folgt, was aus der Wahrheit der induzierten universellen Aussage folgt; aus der Wahrheit dieser universellen Aussage folgt aber die Wahrheit jeder entsprechenden Aussage; diese letzte Wahrheit folgt also auf die Gültigkeit der betreffenden Induktion.

(Ad II.2) Induktion ist ein locus a partibus totius in quantitate ad suum totum; das impliziert Induktion über alle Fälle.

Kommentar: Ein Totum in quantitate ist nach Petrus Hispanus ein Universalbegriff, universell begriffen. Zum Beispiel: omnis homo (jjeder Mensch'), nullus homo ((kein Mensch'). Petrus fügt hinzu, daß ein Locus a toto in quantitate eine Habitudo (‘Relation`) des Ganzen zu seinem Teil ist. Ein Induktion ist darum das Fortschreiten von einem quantitativen Teil (d.h. einigen Einzelfällen) zu seinem quantitativen Ganzen (d.h. allen Fällen). Siehe auch II.2 und die Anmerkung hierzu!

(Ad II.3) Wenn nicht über alle Einzelfälle induziert wird, ist ein logischer Fehler impliziert: Der Vordersatz kann wahr sein, während der Nachsatz falsch ist.

(Ad II.4) Wenn nicht über alle Fälle induziert wird, ist eine Reduktion auf einen Syllogismus unmöglich. 
Kommentar: Siehe III.1.2.

(Ad II.5) Wenn nicht über alle Fälle induziert wird, kann man sich ein Gegenbeispiel denken.

(Ad III) Vier Thesen des Verfassers und die Hauptantwort der Quaestio (Ad III.1) Eine Induktion ist notwendig, wenn über alle Einzelfälle induziert wird.

(Ad III.2) Eine Induktion ist evident, wenn sie sich durch die Hinzunahme einer universellen Prämisse auf einen logisch gültigen Syllogismus reduzieren läßt.

(Ad III.3) Eine Induktion über eine bestimmte Zahl von Einzelfällen, aber nicht über alle genügt, um wahrscheinliche Meinung, Glauben oder Überzeugung herzustellen. Auf diese Weise kommt eine gültige Induktion zustande.

Kommentar: Man muß unterscheiden zwischen einer notwendigen, d.h. logisch notwendigen Induktion, die eine geschlossene Induktion ist; einer evidenten Induktion, d.h. einer notwendigen Induktion, die explizit auf einen Syllogismus reduziert ist; und einer gültigen Induktion, die nur Induktion über eine bestimmte Zahl von Einzelfällen ist.

(Ad III.3. 1-3) Beweis: a. Induktion ist eine stärkere Folgerung als die Argumentationsform des sogenannten Beispiels ${ }^{30}$.

b. Für eine gültige Induktion genügt das, was für die Wahrscheinlichkeit der induzierten universellen Aussage genügt. Für diese genügt aber die Wahrheit einer bestimmten Zahl von singulären Prämissen - vorausgesetzt, daß es kein Gegenbeispiel gibt. Das Ziel einer dialektischen Argumentation ist Überzeugung, nicht Beweis.

Kommentar: Die Zielsetzung einer dialektischen Argumentation, etwas als wahrscheinlich zu erweisen, ist hier verschieden von derjenigen, die oben (im Text I.3) angegeben ist, wo gesagt wird, daß das Ziel eine Prüfung der Konklusion ist. Der anonyme Autor beweist seine These auch aus der sogenannten Obligationspraxis (d.h. Disputationspraxis), bei der ein Respondent eine Induktion akzeptieren muß, wenn er kein $\mathrm{Ge}$ genbeispiel angeben kann.

c. Viele natürliche Prinzipien sind evident aufgrund der Erfahrung. Es ist nicht einfach festzustellen, über wie viele Einzelfälle man induzieren soll: Das hängt von der Materie und dem Abstraktionsgrad ab.

${ }^{30}$ Siehe oben, $\S 2$. 
Kommentar: Meines Erachtens spricht unser Autor hier von einem niedrigeren Grad der Evidenz. Das Annehmen verschiedener Grade der Evidenz findet sich zum Beispiel auch bei Johannes Duns Scotus ${ }^{31}$ und Wilhelm von Ockham ${ }^{32}$. Daß die Zahl der induzierten Einzelfälle auch vom Intellectus' abhängt, soll meines Erachtens heißen, daß der Intellekt einen verschiedenen Abstraktionsgrad hat entsprechend dem Grad der Materie in den Einzeldingen, die der Intellekt betrachtet. Die im Text gegebenen Beispiele von natürlichen Prinzipien sind naturwissenschaftliche Prinzipien.

(Ad III.4) Induktionen zu universellen Aussagen bezüglich akzidenteller Prädikate sind nur über alle Einzelfälle möglich.

Kommentar: Die hier gemeinte Induktion führt zu universellen Aussagen wie ‘Alle Griechen sind weiß) (zufälliges Akzidens) oder vielleicht auch zu ‘Alle Schwäne sind weiß) (sogenanntes universelles Akzidens (vor der Entdeckung schwarzer Schwäne in Australien)).

(Ad III.5) Konklusion

Der Intellekt stimmt freier zu als das Sinnesvermögen, nämlich dann, wenn er aufgrund einer Induktion über eine bestimmte Zahl von Einzelfällen mit einer universellen Aussage übereinstimmt.

Kommentar: Die Interpretation des letzten Satzes ist nicht sicher (ut in mathematicis non sufficeret intellectus). Muß man hier statt (mathematicis: ‘accidentibus, lesen, so daß man übersetzen kann: (wie der Intellekt bei akzidentellen Eigenschaften nicht genügte), d.h. weil er dann nicht auf eine universelle Aussage schließen kann?

(Ad IV) Auseinandersetzung mit den Argumenten zugunsten einer verneinenden Antwort auf die Hauptfrage

${ }^{31}$ Siehe Johannes Duns Scotus, In Sententias, Ordinatio I, distinctio iii, pars v, quaestio iv, in Opera omnia III, liber I, Civitas Vaticana 1954, S. 123-177, bes. S. 138 (n. 229)-148 (n. 145).

${ }^{32}$ Siehe Wilhelm von Ockham, Scriptum in librum primum Sententiarum, Ordinatio, Prologus et distinctio prima (Opera Theologica I), Liber I, Prologus, S. 94-95. Siehe auch Wilhelm von Ockham, Summa logicae, III-2, 10 (Opera Philosophica I), bes. S. 523, Z. 9-21. D. Webering, Theory of demonstration According to William Ockham, 1953 (S. 73-75), ist hier nicht sehr zuverlässig (siehe E.P. Bos, A Contribution to the History of Theories of Induction, in Vorbereitung). 


\section{E. P. Bos}

(Ad IV.1.1) Es ist nicht notwendig, über alle Einzelfälle zu induzieren. Man soll bei einer Induktion über nur wenige Einzeldinge den Ausdruck sund so weiter hinzufügen.

(Ad IV.1.2) Man soll die oben genannten Möglichkeiten (a) und (b) benutzen und unter verschiedener Form induzieren.

(Ad IV.1.3 + IV.1.4): Induktion über eine bestimmte Zahl genügt, wenn es kein Gegenbeispiel gibt.

(Ad IV.2) Auseinandersetzung mit den Argumenten zugunsten einer bejahenden Antwort auf die Hauptfrage

Notwendige oder evidente Induktion muß über alle Einzelfälle erfolgen.

Das Hauptergebnis dieser Quaestio ist, daß Induktion über eine bestimmte Zahl von Einzelfällen für eine wahrscheinliche Erkenntnis genügt und daß mit dieser Erkenntnis naturwissenschaftliche Prinzipien erkannt werden. Eine gültige Induktion, die also nicht notwendig und auch nicht im striktesten Sinne evident ist, d.h. nicht über alle Fälle induziert, führt zu einer wahrscheinlichen Erkenntnis, der nur in einem schwachen Sinne Evidenz zukommt.

Meines Erachtens hat der anonyme Autor einerseits die offene Induktion als fruchtbar für die Wissenschaft erkannt und andererseits gesehen, was aus ihr für die Sicherheit der wissenschaftlichen Prinzipien folgt, daß diese nämlich weniger sicher sind. In dieser Hinsicht kann man diese Theorie mit den älteren Theorien kontrastieren, zum Beispiel mit denjenigen Alberts des Großen und Thomas von Aquins ${ }^{33}$. Diese unterscheiden, was die Gewißheit anbetrifft, zwischen der Naturwissenschaft und Wissenschaften wie der Mathematik unter (qualitativen` Aspekten, wie ich sie oben ${ }^{34}$ genannt habe.

Im Jahre 1906 hat A. Mansion einen Beitrag mit dem Titel <L'Induction chez Albert le Grand ${ }^{35}$ veröffentlicht. Er findet in unserer Quaestio die ersten Spuren einer Theorie wissenschaftlicher (d.h., so Mansion, fruchtbarer) Induktion. Mansions Bemerkungen sind insofern wertvoll, als er darauf hinweist, daß in unserer Quaestio die quantitativen Probleme der Induktion berücksichtigt werden. Leider hat er jedoch einige Fehler gemacht. So schreibt er zum Beispiel den oben abgedruckten Textteil

33 Siehe oben, $\$ 3$.

34 Siehe oben, $\$ 3$.

35 A. Mansion, L'Induction chez Albert le Grand, 1906. 
II dem anonymen Verfasser selbst zu, statt ihn wie einen Textteil aufzufassen, in dem die Ansicht eines Opponenten verteidigt wird. Mansion hat auch nicht gesehen, wie unser Anonymus zwischen notwendiger (eventuell evidentre) und gültiger Induktion unterscheidet ${ }^{36}$, und er meint, ein Prinzip wie romnis ignis est calidus> könne unmöglich ein wissenschaftliches Prinzip sein, sondern nur ein Satz, der zu den alltäglichen Generalisationen gehöre ${ }^{37}$. Dennoch ist das genannte Prinzip nach Duns Scotus, Ockham und auch Buridanus ${ }^{38}$ ein wissenschaftliches Prinzip. Sehr verwirrend ist es, daß Mansion die Theorien des Pseudo-Johannes Duns Scotus und des Johannes Duns Scotus selbst nicht unterscheidet, weil er annimmt, daß alle diese Werke in der Edition Vivès (1889-1895) dem Doctor subtilis zuzuschreiben sind.

Die Theorie des Pseudo-Johannes Duns Scotus, des Autors unserer Quaestio, ist zweifelsohne sehr verschieden von der des Johannes Duns Scotus selbst. Ich kann hier nicht im Detail die Auffassung des Duns Scotus besprechen. Für eine gute Darstellung verweise ich auf Weinberg ${ }^{39}$. Hier genügt es, seine Theorie kurz zusammenzufassen.

Nach Duns Scotus kann der Mensch sich eine sichere, weil evidente, Kenntnis der ersten Prinzipien der Naturwissenschaft erwerben. Obgleich Duns Scotus als Prinzip annimmt, daß alles in der Welt kontingent ist (d.h. abhängig vom Willen Gottes) und daß Gott in die Ordnung der hiesigen Welt immer eingreifen kann, ist Sicherheit der Erkenntnis - anders, als Heinrich von Gent (gestorben 1296) gemeint hat, gegen den Duns Scotus hier polemisiert - möglich, und zwar aufgrund des nach Duns Scotus evidenten Prinzips squicquid evenit ut in pluribus ab aliqua causa non libera, est effectus naturalis illius causae) ('Was meistenfalls geschieht aufgrund einer Ursache, die nicht frei ist, ist die natürliche Folge dieser Ursaches) ${ }^{40}$. Dies zeigt meines Erachtens zur Genüge, daß diese Theorie sich prinzipiell von derjenigen des Pseudo-Johannes Duns Scotus unterscheidet.

36 Ibidem, S. 125.

${ }^{37}$ Ibidem, S. 128.

${ }^{38}$ Siehe unten, $\$ 5$.

${ }^{39} \mathrm{~J}$. Weinberg, Abstraction, Relation and Induction. Three Essays in the History of Thought, 1965, S. 139-142.

${ }^{40}$ Siehe oben, Anm. 31. 


\section{§5. Johannes Buridanus (1300-kurz nach 1358) und Marsilius von Inghen (kurz vor 1340-1396)}

Der Pariser Magister Johannes Buridanus hat in der 20. Quaestio seines Kommentars zu den Analytica Priora unter dem Titel Utrum per inductionem probatur propositio immediata) ( (Wird durch Induktion eine unmittelbare Aussage bewiesen?) unser Thema angesprochen. (Ein vorläufiger Text dieser Quaestio findet sich im Anhang dieses Beitrags). Buridanus unterscheidet zwischen zwei Arten wahrer und unmittelbarer Prinzipien $^{41}: 1$. Prinzipien, die evident sind, weil Subjekts- und Prädikatsterm sich einschließen oder ausschließen, zum Beispiel shomo est animal, ‘nullus homo est asinus ${ }^{42}, 2$. Prinzipien, bei denen es nicht evident ist, daß die gebrauchten Terme sich einschließen oder ausschließen ${ }^{43}$. Zu dieser letzten Art gibt es vier Unterarten, von denen nur die vierte hier relevant ist ${ }^{44}$ : Der Mensch erwirbt sie durch Induktion, zum Beispiel comnis ignis est calidus. Diese Induktion, sagt Buridanus, setzt Sinneswahrnehmung, Erinnerung und Erfahrung voraus. Sie ist keine notwendige Argumentation, aber, wie Buridanus in seiner Quaestio sagt, eine Art guter (d.h. gültiger) Induktion. Durch die naturalis inclinatio intellectus ad verum («die natürliche Neigung des Intellekts zum Wahren') akzeptiert der Intellekt, wenn et sic de singulis (sund so weiter) hinzugefügt worden ist und es kein Gegenbeispiel gibt, die unmittelbare Aussage.

Es scheint mir, daß diese Auffassung von der Rolle der Induktion in der Naturwissenschaft der Theorie des Pseudo-Johannes Duns Scotus sehr nahe steht. Nach Buridanus sind die Prinzipien der Naturwissenschaft weniger sicher als zum Beispiel diejenigen der Mathematik ${ }^{45}$. Er sieht die Rolle des Intellekts als eine Art Schlußstein, der Zustimmung mit einer induzierten universellen Aussage ermöglicht, welche Auffassung sich nicht bei Pseudo-Duns Scotus findet. Marsilius von Inghen (kurz vor 1340-1396) ist in seinem Kommentar (Quaestiones) zu den Analytica Priora

\footnotetext{
41 Siehe das Corpus (\$ III) des Textes im Anhang.

42 Siehe die Edition, § III.1.

43 Siehe die Edition, § III.2.

44 Siehe die Edition, $\$$ III. 4 .

45 Siehe auch A. Maier, Metaphysische Hintergründe der scholastischen Naturphilosophie, 1955, S. 384.
} 
des Aristoteles ${ }^{46}$ in den Hauptpunkten derselben Meinung wie sein Meister in artibus liberalibus Johannes Buridanus.

\section{§6. Schluß}

1. Pseudo-Johannes Duns Scotus entwirft eine Theorie der offenen Induktion. Ob er der erste war, läßt sich hier nicht definitiv feststellen. Diese Argumentationsart ist, wie bekannt, fruchtbar für die Wissenschaft, aber die Unmöglichkeit, über alle Fälle zu induzieren, macht die Prinzipien der Naturwissenschaft nur wahrscheinlich.

2. Die Theorie des Pseudo-Johannes Duns Scotus unterscheidet sich zum Beispiel von der Auffassung Alberts des Großen und Thomas von Aquins; sie unterscheidet sich auch von der des Johannes Duns Scotus; sie steht aber der von Johannes Buridanus und Marsilius von Inghen vertretenen Auffassung nahe und kann daher mit einiger Wahrscheinlichkeit in die erste Hälfte des 14. Jahrhunderts datiert werden.

\section{Bibliographie}

\section{Primärliteratur (lateinische Editionen)}

N.B.: Die Werke des Aristoteles und Averroes sind nicht erwähnt.

Albert der Große, Analytica Priora in B. Alberti Magni (...) Opera omnia (...) cura ac labore A. Borgnet, volumen primum, Parisiis (L. Vivès) 1890

Johannes Duns Scotus, Opera Omnia, I, In Sententias, Ordinatio, Liber primus, distinctio tertia, Civitas Vaticana 1954

Marsilius von Inghen, Quaestiones super libris Priorum Analyticorum, Venetiis 1516 (Reprint Frankfurt am Main (Minerva) 1968)

Petrus Hispanus, Tractatus, called afterwards Summule logicales. First Critical Edition from the Manuscripts with an Introduction by L.M. de Rijk, Assen (Van Gorcum) 1972

Porphyrius, Isagoge, ed. A. Busse, in Commentaria in Aristotelem Graeca, Pars IV (1), 1887, in Porphyrii Isagoge, Translatio Boethii, ed. L. Minio-Paluello, Bruges-Paris (Desclée de Brouwer) 1966

${ }^{46}$ Marsilius von Inghen, Quaestiones super libris Priorum Analyticorum, 1516: liber II, quaestio 20, f. 36rb-vb. 
Thomas von Aquin, In Aristotelis libros Perihermeneias et Posteriorum Analyticorum expositio, cum textu ex recensione Leonina, cura ac studio P. Fr. Raymundi M. Spiazzi, O.P., editio secunda, Augustae Taurini (Marietti) 1955

Thomas von Aquin, Summa Theologiae, I-II, cura et studio P. Caramello (...), Augustae Taurini (Marietti) 1952

Wilhelm von Ockham, Scriptum in librum primum Sententiarum, Ordinatio, prologus et distinctio prima, edidit G. Gál adlaborante S. Brown, St. Bonaventure, N.Y. (St. Bonaventure University) 1967 (Opera Theologica I)

Wilhelm von Ockham, Summa logicae, ed. Ph. Boehner, G. Gál, S. Brown, St. Bonaventure, N.Y. (St. Bonaventure University) 1974 (Opera Philosophica I)

\section{Sekundärliteratur (Studien und Übersetzungen)}

C. Balić, 'The Life and Works of John Duns Scotus', in John Duns Scotus, 12651965, edited by John K. Ryan and Bernardine M. Bonansea, Washington (The Catholic University of America Press) 1965

M. Black, Induction' in The Encyclopedia of Philosophy edited by Paul Edwards, vol. III, New York (The Macmillan Company and The Free Press), London (CollierMacmillan Publishers) 1967: 169-181

E.P. Bos, «A Contribution to the History of Induction, erscheint in: Acts of the 8th European Symposium of Medieval Logic and Semantics (Freiburg im Breisgau), hrsg. K1. Jacobi

A.C.S. McDermott, Notes on the Assertoric and Modal propositional Logic of the Pseudo-Scotus, , History of Philosophy, 10 (1972), pp. 273-306

K. von Fritz, Die Epagoge bei Aristoteles, München (Verlag der bayerischen Akademie der Wissenschaften) 1964 (Bayerische Akademie der Wissenschaften, Philosophisch-Historische Klasse, Sitzungsberichte, Jahrgang 1964, Heft 3)

E. Gilson, Jean Duns Scot. Introduction à ses positions fondamentales, Paris (Vrin) 1952

B. Goelz, sDie echten und unechten Werke des Duns Skotus nach dem gegenwärtigen Stand der Forschung', in Sechste und Siebente Lektorenkonferenz der deutschen Franziskaner, Werl in Westfalen. Sigmaringen 1934, pp. 53-60

M. Grajewski, ‘Duns Scotus in the Light of Modern Research,, in Truth in Contemporary Crisis, Proceedings of the American Catholic Philosophical Association, 1942, pp. 168-185

N.J. Green Pedersen, The Tradition of the Topics in the Middle Ages, The Commentaries on Aristotle's and Boetius' ‘Topics», München-Wien (Philosophia Verlag) 1984.

W. Hess, Erfahrung und Intuition bei Aristoteles' in Phronesis, 15, 1970, pp. 48-82 Ch. Lohr, Medieval Latin Aristotle Commentaries;, in Traditio, Studies in Ancient and Medieval History, Thought and Religion, part 3, 26 (1970), pp. 135-216

A. Maier, Metaphysische Hintergründe der spätscholastischen Naturphilosophie, Rome (Edizioni di Storia e Letteratura) 1955 (Storia e Letteratura, Raccolta di Studi e Testi 52)

A. Mansion, 'L'Induction chez Albert le Grand, in Reoue Néo-scolastique, treizième année, Louvain, 1906, I, pp. 115-134; II, pp. 245-264 
B. Mates, «Pseudo Scotus on the Soundness of Consequentiae` in Contributions to Logic and Method in Honor of J.M. Bochenski, in Logic and methodology, edited by A.T. Tymieniecka in collaboration with C. Parsons, Amsterdam (North Holland Publishing Company) 1965, S. 132-141

A.A. Maurer, St. Thomas Aquinas, The Division and Methods of the Sciences. Questions $V$ and VI of his Commentary on the De trinitate of Boethius translated with Introduction and Notes. Toronto (The Pontifical Institute of Mediaeval Studies), ${ }^{3} 1963$

A.Ch.S. McDermott, «Notes on the Assertoric and Modal Propositional Logic of the Pseudo-Scotus, in Journal of the History of Philosophy, X (1972), S. 273-306.

G. Priest and R. Routley, ‘Lessons from Pseudo-Scotus`, in Philosophical Studies, 42 (1982), S. 189-199.

P. Raymond, 'La théorie de l'induction: Duns Scotus précurseur de Bacon', in Études franciscaines 21 (1909), S. 113-126, 270-279.

S. Read, Self-Reference and Validity, in Synthese, 42 (1979), S. 265-274.

W.D. Ross in: Aristoteles, Prior and Posterior Analytics. A Revised Text with Introduction and Commentary by W.D. Ross, Oxford (at the Clarendon Press) 1949

U. Smeets, Lineamenta bibliographiae Scotisticae, Romae 1942

P. Thom, Conversion of Propositions Containing Singular or Quantified Terms in Pseudo-Scotus' in History and Philosophy of Logic, 3 (1982), S. 129-149

H. Tredennick, in Aristotle, I. Categories, On Interpretation, Prior Analytics, translated by H.P. Cook and H. Tredennick, London (William Heinemann) / Cambridge (Mass.) (Harvard University Press) 1967

N. Tsouyopoulos, ¿Die induktive Methode und das Induktionsproblem in der griechischen Philosophie', in Zeitschrift für allgemeine Wissenschaftstheorie, V/1 (1974), S. 94-122

D. Webering, Theory of Demonstration According to William Ockham, St. Bonaventure (The Franciscan Institute), Louvain (Nauwelaerts), Paderborn (Schöningh) 1953

J. Weinberg, Abstraction, Relation and Induction. Three Essays in the History of Thought, Madison and Milwaukee (The University of Wisconsin Press) 1965 


\section{Anhang}

Johannes Buridanus, Qunestiones super libris Analyticorum Priorum, quaestio XX, aus: MS Leipzig, Universitätsbibliothek, 1372, ff. 119ra-119vb.*

N.B.: add: addidit

del.: delevit

$<\ldots$ : Hinzufügung des Herausgebers

\section{UTRUM PER INDUCTIONEM PROBATUR PROPOSITIO IMMEDIATA}

\section{[Rationes quod non]}

(I.1) Arguitur quod non: quia idem est propositio immediata et principium demonstrationis, ut dicitur primo Posteriorum ${ }^{\mathrm{a}}$; sed principia demonstrationis nulla probatione probantur eo quod debent esse per se nota; ergo propositio immediata nec inductione nec probatione alia probatur.

(I.2). Item, ibi est petitio principii ubi nulla est probatio; sed in inductione petitur principium; ergo et cetera. Probo minorem: quia inductio procedit ex singularibus ad universalem; modo singularia sunt minus nota <universalibus>, non solum simpliciter, sed etiam quoad nos, quoniam in prohemio Physicorumb dicitur quod ab universalibus ${ }^{c}$ ad singularia ${ }^{d}$ oportet procedere, propter hoc quod universalia sunt nobis notiora; ergo inductio procedit ex minus notis et, per consequens, petit principium.

(I.3) Nec immediatum probatur per medium ${ }^{e}$ (hoc est per se notum); sed inductio procedit per medium, cum sit reducibilis ad syllogismum, qui non est sine medio; ergo per inductionem non probatur ${ }^{f}$ immediatum.

[Oppositum]

Oppositum dicit Aristoteles, secundo huiusg.

\footnotetext{
"Ich danke Herrn Professor H. Hubien (Lüttich) für seine Hilfe bei der Edition.

a Aristoteles, Analytica Posteriora, I, II, 72 a 7-8.

b Aristoteles, Physica, I, 184 a 24-25.

' singularibus MS.

d universalia MS.

e mediatum MS.

${ }^{f}$ probat MS.

8 Aristoteles, Analytica Priora, II, xxiii.
} 
(III.1) Notandum <est> quod propositiones verae <et> immediatae sunt principia scientiarum et artium. Sed principia scientiarum et artium sunt duplicia: quaedam sunt manifesta per evidentem inclusionem vel exclusionem terminorum; verbi gratia, quod shomo est animal, , albedo est color, triangulus habet tres angulos aequales duobus rectis?. Si enim (f. $119 \mathrm{rb}$ ) dicitur declaratur quid nominis subiecti, statim apparebit quod dabitur per praedicatum; ideo, scito quid nominis subiecti manifesta est inclusio praedicati in eo; ideo propositio ${ }^{\mathrm{h}}$ est per se nota. Similiter, propter manifestam exclusionem sunt tales per se notae: (nulla albedo est nigredo,, ‘nullus homo est asinus, et cimpossibile est idem simul esse et non esse', et sic de multis aliis. Modo, talia principia per nullam argumentationem probantur vel declarantur, sed omnino supponuntur, quia oportet supponere quid nominis. Quo habito ${ }^{i}$ ista principia sunt evidentia.

(III.2) Alia autem <sunt> principia quorum termini non manifeste et evidenter se includunt vel $\mathrm{j}$ excludunt, tamen dicuntur principias quia sunt indemonstrabilia, <et quia> sine demonstratione <et> sine necessaria consequentia fiunt ${ }^{k}$ nobis evidentia. Et huiusmodi principia fiunt ${ }^{1}$ nobis evidentia aliquo ${ }^{\mathrm{m}}$ quattuor modorum. (III.2.1) Unus modus est per actualem sensum, sicut quod iste ignis est calidus: hoc enim est tibi evidens quando ipsum sentis: similiter quod ${ }^{\text {n }}$ Rubertus scribit $<q u a n d o$ Rubertum vides scribere $>$ et sic de aliis. Et non obstante quod tales propositiones sunt contingentes <et singulares>, et, per consequens, quod non sunt principia in demonstratione, nec intrant scientias demonstrativas, tamen habent locum in artibus $^{\circ}$ et in prudentia, ut manifestatur sexto EthicorumP. Et ideo huiusmodi propositiones singulares, ad sensum evidentes, sunt bene principia ratiocinationum artis et prudentiae.

(III.2.2) Secundo modo huiusmodi principia sunt nobis evidentia per memoriam, ut quod ille ignis erat calidus, et quod Rubertus tunc scribebat. Et adhuc illa principia habent locum in artibus et in prudentia. Saepe enim in moralibus ad praemiandum vel ad puniendum $q$ oportet ratiocinari ex singularibus de praeterito nobis notis ${ }^{r}$ per memoriam.

(III.2.3) Alia <príncipia> sunt nobis nota per experientiam, quae quidem experientia supponit (f. 119 va) sensum et memoriam. <Verbi gratia>, si tu ad sensum co-

h conclusio MS.

${ }^{\mathrm{i}}$ vel add. MS.

jet MS.

k sunt MS.

${ }^{1}$ fiant MS.

m alteri MS.

${ }^{n}$ quando MS.

${ }^{\circ}$ artificibus MS.

P Aristoteles, Ethica Nicomachea, VI, iv und v (besonders 1140 a 12 und 1140 a 32-33).

${ }^{\mathrm{q}}$ praemiendum MS.

${ }^{r}$ notum MS. 
gnovistis quod ignis $\mathrm{A}^{\mathrm{t}}$ erat calidus, et postea idem de igne B, et sic de aliis multis, tu postea videns ignem $C$, et non tangens ipsum ${ }^{u}$, iudicabis ${ }^{v}$ per memoriam $<$ de aliis $>$ et per similitudinem quod ille ignis $\langle\mathrm{C}\rangle$ est calidus; et hoc non est proprie loquendo iudicium sensus, quia non tangis ${ }^{w}$ ipsum, nec solum per memoriam, quia memoria non est nisi ${ }^{x}$ prius cognitorum et tamen istum ignem $C$ numquam alias vidisti necy cognovisti; sed iudicium hoc vocatur sexperimentale. Et non solum homines, immo aequaliter ${ }^{2}$ brutae huiusmodi iudicio utuntur; unde propter hoc canis timet lapidem si aliquis laesit ${ }^{a}$ ipsum. Et omnia praedicta principia sunt singularia, et sunt principia in arte vel in prudentia, et non in scientia demonstrativa vel speculativa.

(III.2.4) Alia principia indigent inductione ad hoc quod fiant evidentia, et iam sunt universalia principia, ut quod omnis ignis est calidus, et quod omne rhebarbarum est purgativum cholerae ${ }^{\mathrm{bb}}$. Ista enim principia fiunt nobis nota per inductionem supponentem sensum, memoriam et experientiam. Cum enim vidisti saepe rhebarbarum purgare choleram ${ }^{\mathrm{cc}}$ et de hoc memoriam habuisti, et quia ${ }^{\mathrm{dd}}$ in multis diversis circumstantiis $\langle h o c\rangle$ consideravisti, numquam tamen ${ }^{\text {ee }}$ invenisti instan-

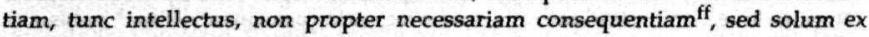
naturali inclinatione eius ad verum, assentit universali principio et capit ipsum tamquam evidens per talem inductionem hoc rebarbarum purgabat $9 g$ choleram <et illud〉, et sic de singulis, quae sensata ${ }^{\text {hh }}$ fuerunt et servata et de quibus memoria ${ }^{\mathrm{ii}}$ habeturij; tunc intellectus supplet istam clausulam ret sic de singulis», eo quod numquam vidit instantiam, licet consideravit in ${ }^{\mathbf{k k}}$ multis circumstantiis, nec ${ }^{11}$ apparet sibi ratio nec ${ }^{\mathrm{mm}}$ dissimilitudo quare debeat esse instantia, et tunc

\footnotetext{
s cognoscis MS.

$\mathrm{t}+\mathrm{n}$ (?) MS.

u et add. MS.

$v_{\text {iudicas MS. }}$

w tangit MS.

${ }^{x}$ non MS.

$y$ vel MS.

$z$ aliqua MS.

aa laedit MS.

bb calore MS.

cc colleram (!) MS.

dd quod MS.

ee enim MS.

ff conclusionem MS.

88 purgat MS.

hh significata MS.

ii memoriam MS.

ij habet MS.

kk de MS.

${ }^{11}$ ex ut ? MS.

$\mathrm{mm}$ vel MS.
} 
concludit universale principium. Et nos bene videmus quod ista non est perfecta probation $^{\text {nn }}$ virtute consequentiae necessariae. Quia omnia quae sensata fuerunt non sufficiunt ad in(f. $119 \mathrm{vb}$ )ferendum universalem conclusionem, quoniam ${ }^{\circ 0}$ praeterPP ista sunt multa alia; et si 19 sufficiunt cum ista clausula cet sic de singulis, tamen ista est accepta per intellectum sine probatione quae sit necessaria consequentia. Et ideo talis universalis propositio vocatur in scientiis demonstrativis non 'conclusio' sed 'principium'; et vocatur 'propositio immediata' quia caret medio $^{\mathrm{rr}}$ per quod ${ }^{\text {ss }}$ possit probari illatione necessaria. Et sic habemus declaratum quo modo per inductionem et per naturalem inclinationem ${ }^{\text {th }}$ intellectus ad verum probatur propositio immediata.

\section{[Ad rationes]}

Et per hoc solvendae sunt rationes.

(IV.1) Ad primam dicendum est quod principium demonstrationis non demonstrabile est; tamen probari potest, sive fieri evidens, per experimentalem inductionem modo dicto.

(IV.2) Ad aliam dicendum est quod in prohemio Physicorum uu dicebat Aristoteles quod universalia sunt nobis notiora singularibus, sed loquebatur, ut dicit Commentator ${ }^{v v}$, non de singularibus simpliciter, sed de singularibus respective; de istis hoc est dictum quod magis universalia sunt nobis notiora minus ${ }^{\text {ww }}$ universalibus, tamen singularia ${ }^{x x}$ sensata sunt nobis notiora.

(IV.3) Ad aliam <dicendum est> quod in inductione est bene medium distinctumyy ab extremitatibus conclusionis, sed non est ibi medium innatum facere evidentiam per consequentiam necessariam, et hoc in illis inductionibus per quas probantur principia demonstrationum. Unde si reducuntur ad syllogismum necessariae consequentiae, tunc ${ }^{22}$ ibi alia propositio accipitur ${ }^{\text {aaa }}$, quae non est manifesta nisi per naturalem inclinationem intellectus ad verum, qui in similibus capit ita esse sicut visum est in aliis.

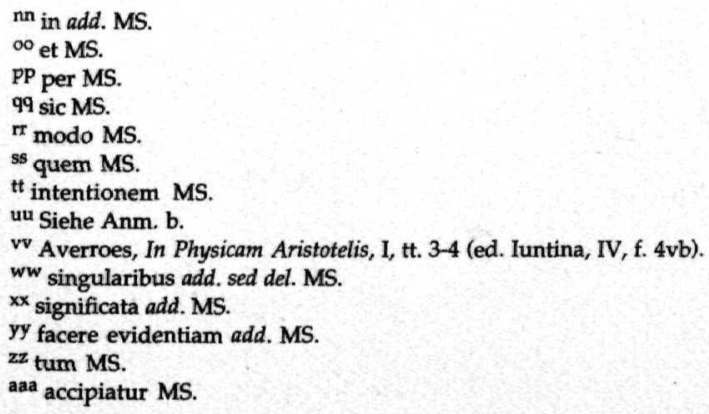

